\title{
Numerical Solution of the Differential Equation for Simulation of the Rice Blast Disease
}

\section{${ }^{1}$ SITTISAK, I; ${ }^{1}$ SARUDA, H; ${ }^{1}$ *ANGKOOL, W; ${ }^{2}$ THIDARAT, B; ${ }^{1}$ USA, H}

\author{
${ }^{1}$ Department of Mathematics, Faculty of Science \\ King Mongkut's University of Technology Thonburi, Bangkok 10140, Thailand \\ ${ }^{2}$ Center of Excellence on Hazardous Substance Management, Department of Environmental Engineering, Faculty of Engineering, King \\ Mongkuts University of Technology Thonburi, Bangkok 10140, Thailand \\ *Email: angkool.wan@kmutt.ac.th
}

\begin{abstract}
Rice blast disease is one of the diseases that causes damage to rice yield in Thailand. This research aims to simulate the severity of rice blast disease using the EPIRICE model for Khao Dawk Mali 105 that caused by Pyricularia oryzae in Prachin Buri, Thailand, and evaluate the simulation results by comparing with field collection data using root mean square error (RMSE). The epidemiological model consists of a system of ordinary differential equations (ODEs) that describes the dynamic of rice leaf disease. The Euler's method is used to approximate the solutions of the ODEs. According to the RMSE, the simulation results were good agreement with the field collection data. Therefore, the numerical methods can be the technical tool for solving the severity of rice blast disease.
\end{abstract}

\section{DOI: https://dx.doi.org/10.4314/jasem.v21i7.8}

Copyright (C) 2017 Sittisak et al. This is an open access article distributed under the Creative Commons Attribution License (CCL), which permits unrestricted use, distribution, and reproduction in any medium, provided the original work is properly cited

Received 16 July 2017; received in revised form 22 September 2017; accepted 10 December 2017

Keywords: EPIRICE model, Khao Dawk Mali 105, Numerical Solution, Pyicularia oryzae, Rice blast disease.

Thailand has a strong tradition of rice production. It has the fifth-largest amount of land under rice cultivation in the world and is the world's second largest exporter of rice. Rice cultivar which brings income to the country is Khao Dawk Mali 105 (KDML105). The advantages of KDML105 are soft and contain a natural fragrant aroma. Although KDML105 is the international market demand, the quantity of rice is not enough to be supplied (Watanesk et al., 2010). The major factor that affects the decreasing of rice crop is the outbreaks of rice blast disease that can damage the rice plant through all stages of growth in high intensity level. In Thailand, the disease dispersal in 1992 damaged over 650,000 tons or $60 \%$ of the total rice yield.

The blast epidemic was reported with total area damaged 2,000,840,000 $\mathrm{m}^{2}$ in north and northeast of Thailand, causing serious economic loss (Disthaporn, 1994). In 2001, it was found that the outbreak of rice blast disease was more than $126,044,800 \mathrm{~m}^{2}$. In 2010, it was found that the outbreak of rice blast disease in Maha Sarakham, Ubon Ratchathani and Buriram provinces from September to December, the damaged area was over $1,269,120,000 \mathrm{~m}^{2}$ (Khanthong et al., 2010). The outbreak of rice blast disease covered the eastern area of Thailand especially in Prachin Buri, Chachoengsao and Nakhon Nayok provinces from 2011 to 2013 (Janlapha et al., 2014).

The mathematical model has been used to describe the epidemic dynamics of the rice blast disease. Kim et al. (2015) studied a quantitative analysis of two rice diseases, including leaf blast and sheath blight that result in the greatest damages using the generic epidemiological model, EPIRICE. The EPIRICE was used to interpret the magnitude of changes in disease risk and to estimate climate change impacts on disease losses and disease control. Therefore, this study aims to simulate the severity of KDML105 rice blast disease under the tropical climate conditions using rice disease epidemiological model in Prachin Buri, Thailand.

Model of rice blast disease: The generic model for rice disease is an EPIRICE model that simulates potential epidemics in a susceptible variety. The EPIRICE model is a simple SEIR model (Segarra et al., 2001). It was a model framework for fungi, viruses and bacteria. The model can be used to describe biological interactions of the rice disease (Savary et al., 2012). The system considered is $1 \mathrm{~m}^{2}$ of a rice crop stand, and epidemics are simulated over a 77-day duration using a daily time step (Kim et al., 2015).

Two components have been added to the structure compose of the senescence of plant tissues and crop growth. It involves four state variables of a crop stand as healthy (H), latent (L) (Roumen and Boef, 1993), infectious (I) (Magboul, et al., 1992), and postinfectious sites (R). An epidemic is the progress of disease in time and space (van Maanen and $\mathrm{Xu}$, 2003). The EPIRICE model consists of 2 modules: the site growth senescence module and SEIR module.

The site growth senescence module is a normal growth cycle, from the seedlings to heading and senescence to life expectancy. The other module is the rice disease which based on SEIR model. When 
the healthy site has pathogen into the latent stage, the symptoms appeared in the infectious stage, after that the rice moved to the removed stage.

The rate of change in each state over time can be described by mathematical equations. The whole model of rice blast disease is given by the following system of ordinary differential equations (ODEs):

$$
\begin{aligned}
& \frac{d H}{d t}=R_{R G} H\left(1-\frac{T_{S}}{S_{X}}\right)-\left(R_{R}+R_{R S} H\right)-R_{C} I\left(\frac{H}{T_{S}}\right) \\
& \frac{d S}{d t}=R_{R}+R_{R S} H \\
& \frac{d L}{d t}=R_{C} I\left(\frac{H}{T_{S}}\right)-\frac{L}{p} \\
& \frac{d I}{d t}=\frac{L}{p}-\frac{I}{i} \\
& \frac{d R}{d t}=\frac{I}{i}
\end{aligned}
$$

where $R_{R G}$ is the relative rate of growth, $H$ is the number of healthy sites, $S_{X}$ is the maximum number of sites a crop stand can produce, $T_{S}$ is the total number of sites, $S$ is the number of senescence sites , $R_{R}$ is the rate of removal, $R_{R S}$ is an intrinsic rate of ageing of healthy tissues or a rate of removal of healthy sites , $I$ is the number of infectious sites, $R_{C}$ is the number of daily new infections originating from existing, $L$ is the number of latent sites, $p$ is period of the latent state, and $i$ is period of the infectious stage.

Numerical solutions of rice blast disease: In this work, the rice blast disease can be described by the rate of rice change in each state (healthy, senescence, latent, infectious and removed site) over time. Moreover, the solution of differential equation is the number of each sites, which is solved by Euler's method. In mathematics, the Euler's method is a firstorder numerical procedure for solving ODEs with a given initial value. It is the most basic explicit method for numerical integration of ODEs.

\section{The number of healthy sites}

$$
\begin{aligned}
& \text { From } \frac{d H}{d t}= \\
& R_{R G} H\left(1-\frac{T_{S}}{S_{X}}\right)-\left(R_{R}+R_{R S} H\right)-R_{C} I\left(\frac{H}{T_{S}}\right),
\end{aligned}
$$

the number of healthy site is solved by the Euler's method as

$$
\begin{aligned}
& \frac{H_{t+1}-H_{t}}{\Delta t}= \\
& \left(R_{R G} H\left(1-\frac{T_{S}}{S_{X}}\right)-\left(R_{R}+R_{R S} H\right)-R_{C} I\left(\frac{H}{T_{S}}\right)\right)_{t}
\end{aligned}
$$

and therefore, the number of healthy site is $H_{t+1}=H_{t}+\left(R_{G}-R_{S}-R_{I}\right)_{t} \Delta t, \quad$ where $R_{G} \equiv R_{R G} H\left(1-\frac{T_{S}}{S_{X}}\right), \quad R_{S} \equiv\left(R_{R}+R_{R S} H\right)$ and $R_{I} \equiv R_{C} I\left(\frac{H}{T_{S}}\right)$

The number of senescence sites

From $\frac{d S}{d t}=R_{R}+R_{R S} H$,

the number of senescence site is solved by the Euler's method as

$\frac{S_{t+1}-S_{t}}{\Delta t}=\left(R_{R}+R_{R S} H\right)_{t}$,

and therefore, the number of senescence site is

$S_{t+1}=S_{t}+\left(R_{S}\right)_{t} \Delta t$, where

$R_{S} \equiv\left(R_{R}+R_{R S} H\right)$.

The number of latent sites

From $\frac{d L}{d t}=R_{C} I C-\frac{L}{p}$,

the number of latent site is solved by the Euler's method as

$\frac{L_{t+1}-L_{t}}{\Delta t}=\left(R_{C} I C-\frac{L}{p}\right)_{t}$,

and therefore, the number of latent site is

$L_{t+1}=L_{t}+\left(R_{I}-R_{T}\right)_{t} \Delta t$, where $R_{I} \equiv R_{C} I C$,

and $R_{T} \equiv \frac{L}{p}$.

The number of infectious sites

From $\frac{d I}{d t}=\frac{L}{p}-\frac{I}{i}$,

the number of infectious site is solved by the Euler's method as

$$
\frac{I_{t+1}-I_{t}}{\Delta t}=\left(\frac{L}{p}-\frac{I}{i}\right)_{t},
$$


and therefore, the number of infection site is

$$
\begin{aligned}
& I_{t+1}=I_{t}+\left(R_{T}-R_{R}\right)_{t} \Delta t, \text { where } R_{T} \equiv \frac{L}{p}, \text { and } \\
& R_{R} \equiv \frac{I}{i} .
\end{aligned}
$$

The number of removed sites

$$
\text { From } \frac{d R}{d t}=\frac{I}{i},
$$

the number of removed site is solved by the Euler's method as

$$
\frac{R_{t+1}-R_{t}}{\Delta t}=\left(\frac{I}{i}\right)_{t},
$$

and therefore, the number of removed site is

$$
R_{t+1}=R_{t}+\left(R_{R}\right)_{t} \Delta t \text {, where } R_{R} \equiv \frac{I}{i} \text {. }
$$

Thus, the whole model is given by the following system of equations:

$$
\begin{aligned}
H_{t+1} & =H_{t}+\left(R_{G}-R_{S}-R_{I}\right)_{t} \Delta t, \\
S_{t+1} & =S_{t}+\left(R_{S}\right)_{t} \Delta t, \\
L_{t+1} & =L_{t}+\left(R_{I}-R_{T}\right)_{t} \Delta t, \\
I_{t+1} & =I_{t}+\left(R_{T}-R_{R}\right)_{t} \Delta t, \\
R_{t+1} & =R_{t}+\left(R_{R}\right)_{t} \Delta t .
\end{aligned}
$$

By solving equations (2), we get the number of each site. The disease severity is measured by the disease leaf area over the whole area via the following formula

$$
y=\frac{D-R}{T_{S}-R} \times 100,
$$

where $y$ is percentage of a disease severity, $D$ is total number of disease sites, $R$ is total number of removed sites, and $T_{S}$ is the total number of sites. The EPIRICE model uses historical incidence data and fine scale weather data in Prachin Buri, Thailand. The meteorological input variables of the model are consisting of date, temperature, relative humidity and rainfall. The output of the model was based on changes in disease severity. The result of model can be used to simulate disease severity about growing season from August 2015 to July 2016.

The simulation of rice blast disease: The EPIRICE model which is translated to R language was used to simulate the severity of rice blast disease. The severity of rice blast disease is calculated for 10 fields, and the result of the EPIRICE model was evaluated with observed data using the RMSE method. The rice plant of first, second, third, fourth and fifth fields were on August, September, October, November and December in 2015, respectively. The sixth, seventh, eighth, ninth and tenth fields were on January, February, March, April and May in 2016, respectively. The severity of rice blast disease of each field in the Prachin Buri was shown in Figure 1 (A-F). The patterns of simulated disease severity correspond to the patterns of observed data. The simulation started early 17 days after planting because the rate of infection started around 15 days after planting. The simulated disease severity was lower or higher than the observed severity data. In the first field, the observed data shows that the outbreak of rice blast disease was occurring 20 to 65 days after planting which the severity is about 1,5 and 25 percent of disease. The simulated data shows that the outbreak of rice blast disease was occurring 17 to 110 days after planting (Figure.1A). In the second, third and fourth fields (figure not shown), the outbreak of rice blast disease did not occur in these fields, because some environment conditions were not favorable for disease outbreaks. The disease severity of fifth field (figure not shown) was occurring about 90 percent of disease and finally rice died at the end of season. In the sixth field, the shape of simulation looked like the observation. The maximum severity of simulation was lower than the observed data (Figure .1B). The outbreak of rice blast disease in the seventh field was occurring about 1 percent of disease and then recovered from the disease. So it conforms to the simulated data (Figure .1C). In the eighth field, the maximum disease severity of observation was 50 percent of disease. But the maximum severity of simulation was around 3 percent of the disease (Figure .1D) since the season of rice planting had high temperature. In fact, it was unfavorable for the disease outbreak. In the ninth field, the disease severity was around 1 percent of the disease. The simulation had the same shape with observed data (Figure 1E) since the conditions were unfavorable for disease outbreaks, for example, the relative humidity was less than 90 percent. In the tenth field, the simulated disease severity was underestimated the observed severity data during the outbreak of the disease (Figure $.1 \mathrm{~F}$ ).
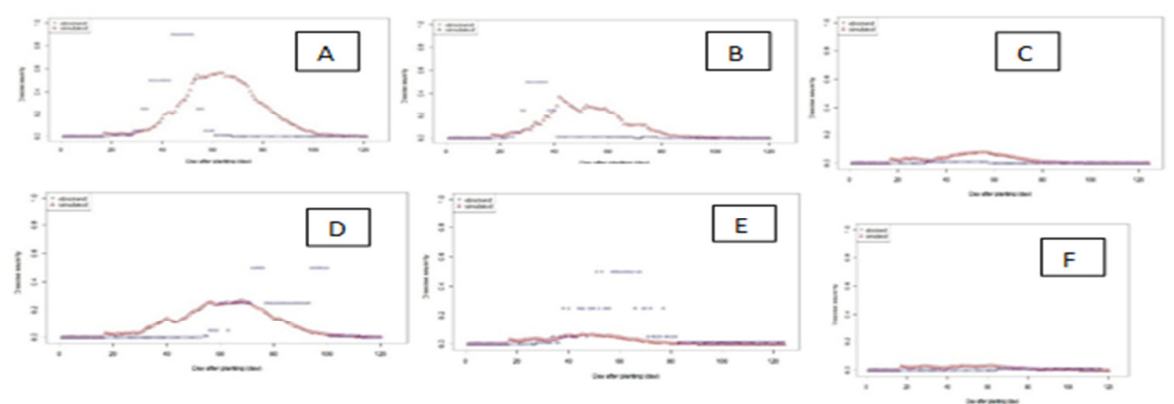

Fig 1: Graphical comparisons of observed (blue dot) and simulated (red dot) disease severity for rice blast disease in the first (A), sixth (B), seventh (C), eighth (D), ninth (E) and tenth (F) fields. 
Table: 1 The statistical comparison of the disease severity from the EPIRICE model with the observed data

\begin{tabular}{ccccccc}
\hline Field No. & $\mathbf{1}$ & $\mathbf{6}$ & $\mathbf{7}$ & $\mathbf{8}$ & $\mathbf{9}$ & $\mathbf{1 0}$ \\
\hline RMSE & 0.2979 & 0.1683 & 0.0327 & 0.1744 & 0.0131 & 0.1608 \\
\hline
\end{tabular}

The performance evaluation of the model: To evaluate the performance of the EPIRICE model between simulation and observation data in 10 fields, the RMSE was used to measure the difference between values simulated by a model and the values observed from the environment. The statistical comparison of the disease severity from the EPIRICE model with the observed data was shown in Table 1. It is found that the RMSE was small and was between 0.0131 and 0.2979 . The RMSE value of the seventh and ninth field were 0.0327 and 0.0131 respectively, which tends to 0 . It means that the model has accuracy in those fields.

Conclusion: The EPIRICE model is a model that can be used to simulate the outbreak of rice. By comparison, the field collection data was higher than the simulation and there were still discrepancies. The reason for the discrepancy is the effect of weather variables on crop dynamics and the effects of other aspects of environmental change. In the seventh and ninth fields, the RMSE tends to 0, which means that the model has an accuracy in those fields. The preliminary results showed that the EPIRICE model can simulate the severity of rice blast disease for KDM 105 in Thailand.

Acknowledgment: The authors would like to thank the Prachin Buri Rice Research Center for historical incidence data of rice blast disease. This research was partially supported by the Faculty of Science, King Mongkut's University of Technology Thonburi. Finally, the authors sincerely thank the financial support provided by King Mongkut's University Thonburi through the "KMUTT $55^{\text {th }}$ Anniversary Commemorative Fund".

\section{REFERENCES}

Disthaporn, S (1994). Current rice blast epidemics and their management in Thailand. In: Zeigler RS, Leong SA, Teng, PS (eds) Rice blast disease, IRRI, The Philippines, p. 333-341.

Janlapha, W; Katenate, T; Noenplab, A; Seewisut, S; Chalermpolyotin, C; Wattanasuchat, R (2014). The Effects of Climate Change on Rice Disease Epidemics in the Eastern Area. Library of Bureau of Rice Research and Development. Rice Department. p. 268.
Khanthong, S; Jantasuriyarat, C; Katengam, S (2010). Blast disease and rice breeding for blast disease resistance through marker-assisted selection, Thai Journal of Genetics. 3(2): 106-119.

Kim, KH; Cho, J; Lee, YH; Lee, WS (2015). Predicting potential epidemics of rice leaf blast and sheath blight in South Korea under the RCP 4.5 and RCP 8.5 climate change scenarios using a rice disease epidemiology model, EPIRICE. J.Agricultural and Forest Meteorology. 203: 191-207.

Magboul, AM; Geng, S; Gilchrist, DG; Jackson, LF (1992). Environmental Influence on the Infection of Wheat by Mycosphaerella graminicola. J. The American Phytopathological Society. 82: 14071413.

Roumen, EC; and de Boef, WS (1993). Latent period to leaf blast in rice and its importance as a component of partial resistance. J. Euphytica. 69: 185-190.

Savary, S; Nelson, A; Willocquet, L; Pangga, I; Aunario, J (2012). Modeling and mapping potential epidemics of rice diseases globally. $J$. Crop Protection. 34: 6-17.

Segarra, J; Jeger, MJ; van den Bosch, F (2001). Epidemic Dynamics and Patterns of Plant Disease. J. The American Phytopathological Society. 91: 1001-1010.

Van Maanen, A; Xu, XM (2003). Modelling plant disease epidemics. J. Plant Pathol. 109: 669-682.

Watanesk, O; Prasertsak, A; Taprab, S; Cheaupan, K; Chamaruak, V; Naklang, K; Wongpiyachon, S; Warintarak, B; Mekwatanakarn, P; Chaidee, S (2010). Khao Dawk Mali 105. Rice Department. Bureau of Rice Research and Development, Bangkok, Thailand. 